\title{
(10)
}

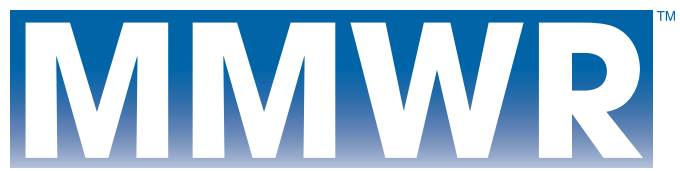

\author{
Early Release \\ Vol. 55 / February 3, 2006
}

Morbidity and Mortality Weekly Report

\section{New Laboratory Assay for Diagnostic Testing of Avian Influenza A/H5 (Asian Lineage)}

On February 3, 2006, the Food and Drug Administration (FDA) announced clearance of the Influenza A/H5 (Asian Lineage) Virus Real-Time Reverse TranscriptionPolymerase Chain Reaction (RT-PCR) Primer and Probe Set and inactivated virus as a source of positive RNA control for the in vitro qualitative detection of highly pathogenic influenza $\mathrm{A} / \mathrm{H} 5$ virus (Asian lineage). Two genetic lineages of influenza $\mathrm{A} / \mathrm{H} 5$ viruses exist: Eurasian (Asian) and North American. The primer and probe set, developed at $\mathrm{CDC}$, is designed to detect highly pathogenic influenza $\mathrm{A} / \mathrm{H} 5$ viruses from the Asian lineage associated with recent laboratory-confirmed infections of avian influenza in humans in east Asia and, most recently, in Turkey and Iraq.

From December 1, 2003, through February 3, 2006, the World Health Organization (WHO) reported 161 confirmed human cases of avian influenza A (H5N1); of these, $86(53 \%)$ were fatal (1). The infections occurred in Cambodia, China, Indonesia, Iraq, Thailand, Turkey, and Vietnam. No infections with avian influenza A/H5 (Asian lineage) have been reported in animals or humans in North America. Since February 2004, CDC has recommended enhanced surveillance in the United States for possible cases of human infection with avian influenza A (H5N1) virus (2). Consistent with these interim recommendations, testing for this virus is indicated when a patient has symptoms of severe respiratory illness and a risk for exposure (e.g., direct contact with ill, dead, or infected poultry in a country with outbreaks of influenza $\mathrm{H} 5 \mathrm{~N} 1$ among poultry). Testing for influenza A/H5 (Asian lineage) should be considered on a case-by-case basis in consultation with local or state health departments.

Testing with the FDA-cleared laboratory RT-PCR assay should be conducted in conjunction with other laboratory testing and clinical observations to help diagnose influenza in patients who might be infected with influenza $\mathrm{A} / \mathrm{H} 5$ (Asian lineage) viruses and to provide epidemiologic information for surveillance purposes. The test also will help to identify influenza A/H5 (Asian lineage) viruses in laboratory viral cultures. Definitive diagnosis of influenza $\mathrm{A} / \mathrm{H} 5$ (Asian lineage), either directly from patient specimens or from viral culture, might require additional laboratory testing and clinical and epidemiologic assessment in consultation with national influenza surveillance experts. Negative results do not preclude influenza virus infection and should not be used as the sole basis for treatment or other patient management decisions.

Testing with the new assay will be limited to laboratories designated by the Laboratory Response Network (LRN), which consists of approximately 140 U.S. laboratories in 50 states. LRN-designated laboratories ensure that the laboratory employs experienced personnel who 1) are trained in standardized rapid molecular procedures, 2) perform analyses in facilities with appropriate biosafety equipment and containment procedures, and 3) use established means for communication with public health programs. Influenza A/H5 (Asian lineage) assay protocols and reagents will be distributed by CDC to designated LRN laboratories nationwide during the week of February 6-10, 2006. The real-time RT-PCR primer and probe set is the only laboratory method that has been cleared by FDA for avian influenza $\mathrm{A} / \mathrm{H} 5$ (Asian lineage) testing and in vitro diagnostic medical device use in the United States. Additional information about the laboratory assay is available at http://www.fda.gov.

\section{References}

1. World Health Organization. Cumulative number of confirmed human cases of avian influenza A/(H5N1) reported to WHO. Geneva, Switzerland: World Health Organization; 2006. Available at http://www. who.int/csr/disease/avian_influenza/en.

2. US Department of Health and Human Services. HHS pandemic influenza plan. Supplement 2: laboratory diagnostics, appendix 2. Interim recommendations: enhanced U.S. surveillance and diagnostic evaluation to identify cases of human infection with avian influenza A (H5N1). Washington, DC: US Department of Health and Human Services. Available at http://www.hhs.gov/pandemicflu/plan/sup2.html\#app2. 